\title{
PERSPECTIVA SOBRE O ENTENDIMENTO DO CUIDADO FARMACÊUTICO AO IDOSO EM UMA INSTITUIÇÃO FILANTRÓPICA
}

\author{
PERSPECTIVE ON THE UNDERSTANDING OF \\ PHARMACEUTICAL CARE TO THE ELDERLY IN \\ A PHILANTHROPIC INSTITUTION
}

\begin{abstract}
Hérick Hebert da Silva Alves', Sanny Ellen de Souza Pereira', Sandna Larissa Freitas dos Santos', Karla Bruna Nogueira Torres Barros ${ }^{2}$, Maria Luísa Bezerra de Macedo Arraes ${ }^{3}$
\end{abstract}

\section{RESUMO}

Objetivo: Apresentar a perspectiva do idoso quanto a atenção farmacêutica. Metodologia: Estudo descritivo, transversal, com uma abordagem semi-quantitativa, realizado na casa de acolhida Remanso da Paz, no município de Quixadá-CE em agosto de 2016. Resultados: Foram avaliados 21 idosos de ambos os sexos, realizando atividades de educação e saúde, através do projeto de extensão Serviço de Atendimento Farmacêutico ao Idoso - SAFI. Dos idosos estudados, houve maior predominância de mulheres 13 (62\%), viúvos 13 (62\%), casa própria 15 (72\%), com faixa etária entre 67 a 91 anos de idade e quanto a escolaridade $11(56 \%)$ tinham o $1^{\circ}$ grau completo. $17(81 \%)$ relataram que o projeto vem influenciando e as orientações e palestras que são ministradas, contribuíram na vida de $18(86 \%)$ dos idosos, onde 16 (76\%) fazem o uso dos conhecimentos adquiridos em seu cotidiano, 3 (14\%) afirmavam conhecer a expressão "Interação medicamentosa", 7 (33\%) "Sobredosagem" e "Automedicação". Portanto, 19 (90\%) afirmaram utilizar seus medicamentos de forma correta. Conclusão: Observa-se a intervenção positiva da atuação do projeto SAFI na vida dos idosos estudados.

Descritores: Saúde do Idoso; Automedicação; Atenção Farmacêutica.
${ }^{1}$ Acadêmico(a) de Farmácia no Centro Universitário Católica de Quixadá (Unicatólica), Quixadá, Ceará, Brasil.

${ }^{2}$ Mestre em Ensino na Saúde pela Universidade Estadual do Ceará (UECE), Fortaleza, Ceará, Brasil.

${ }^{3}$ Mestre em Patologia pela Universidade Federal do Ceará (UFC), Fortaleza, Ceará, Brasil.

Objective: To present the perspective of the elderly regarding pharmaceutical care. Methodology: A descriptive, cross-sectional study with a semi-quantitative approach, carried out at the Remanso da Paz shelter in the municipality of Quixadá-CE in August 2016. Results: Twenty-one elderly men and women were evaluated, performing education and Health, through the project of extension Service of Pharmaceutical Care to the Elderly - SAFI. Among the elderly studied, 13 (62\%), 13 (62\%), 15 (72\%), $67-91$ years of age, and $11(56 \%)$ had The complete 1 st grade. 17 (81\%) reported that the project has been influencing and the guidelines and lectures given have contributed to the life of $18(86 \%)$ of the elderly, where $16(76 \%)$ use the knowledge acquired in their daily lives, $3(14 \%)$ claimed to know the expression "Drug Interaction", 7 (33\%) "Overdose" and "Self-medication". Therefore, 19 (90\%) stated that they used their medications correctly. Conclusion: The positive intervention of the SAFI project in the life of the elderly studied is observed.

Descriptors: Health of the Elderly; Self Medication; Pharmaceutical Care. 


\section{Introdução}

O processo de envelhecimento populacional vem sendo alvo de atenção na maioria dos países, como uma resposta à mudança de alguns indicadores de saúde, especialmente a queda da fecundidade e da mortalidade e o aumento da esperança de vida'.

O cuidado ao idoso deve primeiramente surgir da família, porém quando esta falha em seu papel protetivo, o poder público e a sociedade, devem solidariamente desempenhar esta atribuição, devendo assegurar ao idoso, a efetivação do direito à vida, à saúde, à educação, ao lazer, ao respeito e à convivência familiar e comunitária. Atualmente sabe-se que em muitos casos, os idosos passam por um processo de solidão, em virtude das alterações que ocorrem na família, aumentando o número de pessoas maiores de sessenta e cinco anos de idade vivendo sozinhas, ou em casas de apoio².

A terceira idade é a fase da vida que requer uma atenção especial, pois é nesta etapa da vida em que ocorrem várias mudanças fisiológicas, colocando a maioria dos idosos em estado de sedentarismo e possibilitando o aumento de doenças crônicas. $O$ uso de medicamentos, por serem muitas vezes deixado de lado pelos seus familiares e não ter acompanhamento em casa, muitos dos idosos não sabem a forma correta de tomar os medicamentos. Para o idoso, o aconselhamento acerca do uso racional de medicamento é essencial, em função da presença frequente de múltiplas patologias, requerendo terapias diferentes, as quais podem resultar no uso concomitante de vários medicamentos. Desse modo, torna-se necessária uma estratégia de administração que diminua os riscos de efeitos colaterais ou adversos e de interações medicamentosas ${ }^{3,4}$.

Com isso, a intervenção do farmacêutico se torna importante, pois é um profissional que, além das habilidades humanísticas, detém os conhecimentos sobre medicamentos, e poderá orientar tanto o paciente quanto o familiar ou acompanhante em relação ao uso racional dos medicamentos, fazendo-os compreender desde a sua prescrição até as orientações quanto ao uso e possíveis interações ${ }^{5}$. A pesquisa teve como objetivo, apresentar a perspectiva do idoso em relação ao projeto de extensão acadêmica Serviço de Atendimento Farmacêutico ao Idoso (SAFI), da instituição de ensino Centro Universitário Católica de Quixadá (Unicatólica), desenvolvendo um levantamento sobre a mudança dos hábitos diários, em busca da prevenção de problemas relacionados à automedicação e o conhecimento a respeito da atuação do profissional farmacêutico no cuidado ao idoso.

\section{Metodologia}

Foi realizado um estudo descritivo, transversal, consistindo em uma abordagem semi-quantitativa, na casa de acolhida aos idosos Remanso da Paz, no município de Quixadá-CE no mês de agosto de 2016, que possui assistência de profissionais médicos, fisioterapeutas, nutricionistas, enfermeiros, e de serviços gerais. A instituição filantrópica conta com doações para manter suas ações realizando atividades ocupacionais e educativas, sendo mediadas por profissionais voluntários.

Foram entrevistados 21 idosos de ambos os sexos, que se encontravam na instituição no momento da pesquisa. Além disso, foram desenvolvidas atividades de educação e saúde, semanalmente, abordando assuntos sobre o uso de medicamentos, reações adversas, interações medicamentosas, plantas medicinais e doenças crônicas, inseridas no âmbito das perspectivas do projeto de extensão Serviço de Atendimento Farmacêutico ao Idoso - SAFI, da instituição de ensino Centro Universitário Católica de Quixadá (Unicatólica) ${ }^{6}$. As atividades do projeto são ministradas por acadêmicos do curso de Farmácia, em linguagem adaptadas aos idosos, para facilitar o entendimento e vincular a teoria abordada em sala de aula com a vivência no âmbito da profissão.

Os dados foram inseridos no banco de dados do software Microsoft Excel para viabilizar o processamento e análise das respostas obtidas. A abordagem quantitativa foi avaliada pelo método de SPSS e a qualitativa pelo método de Bardin 7.0 presente estudo foi aprovado pelo Comitê de Ética do Centro Universitário Católica de Quixadá com o parecer 1.659.170, através da Plataforma Brasil de acordo com a Resolução 466/12 do Conselho Nacional de Saúde, que regulamenta as diretrizes e normas da pesquisa em seres humanos ${ }^{8}$, seguindo as determinações desta que são especificidades das pesquisas com seres humanos. 


\section{Resultados}

A identificação dos idosos participantes está descrita na tabela 1. No total $13(62 \%)$ eram mulheres e $8(38 \%)$ homens, com faixa etária entre 67 a 91 anos de idade.

Tabela 1 - Características sociodemográficas dos idosos estudados, Quixadá (CE), 2016.

\begin{tabular}{|c|c|c|}
\hline \multicolumn{3}{|c|}{ Características sócio econômicas } \\
\hline Idade & Frequência & $\%$ \\
\hline 67 a 72 & 10 & $48 \%$ \\
\hline 73 a 80 & 6 & $28 \%$ \\
\hline 81 a 91 & 5 & $24 \%$ \\
\hline \multicolumn{3}{|l|}{ Escolaridade } \\
\hline $1^{\circ}$ grau completo & 11 & $53 \%$ \\
\hline $2^{\circ}$ grau completo & 6 & $28 \%$ \\
\hline Não alfabetizado & 4 & $19 \%$ \\
\hline \multicolumn{3}{|l|}{ Estado civil } \\
\hline Viúvos & 13 & $62 \%$ \\
\hline Casados & 5 & $24 \%$ \\
\hline Solteiro & 2 & $9 \%$ \\
\hline Separados & 1 & $5 \%$ \\
\hline \multicolumn{3}{|c|}{ Situação Habitacional } \\
\hline Própria & 15 & $72 \%$ \\
\hline Familiares & 3 & $14 \%$ \\
\hline Alugada & 2 & $9 \%$ \\
\hline Cedida & 1 & $5 \%$ \\
\hline
\end{tabular}

Antes da implantação do projeto de extensão SAFI (Gráfico 1), 3 (33\%) dos idosos fazia automedicação, porém, apenas 5 (24\%) conheciam os efeitos dos medicamentos que eles utilizavam e logo após a implantação 19 (90\%) deles parou a automedicação mediante todas as informações que foram abordadas nos encontros com palestras e orientações sobre o risco da automedicação.

Gráfico 01 - Intervenção anterior e posterior ao Projeto SAFI dos idosos estudados, Quixadá (CE), 2016.
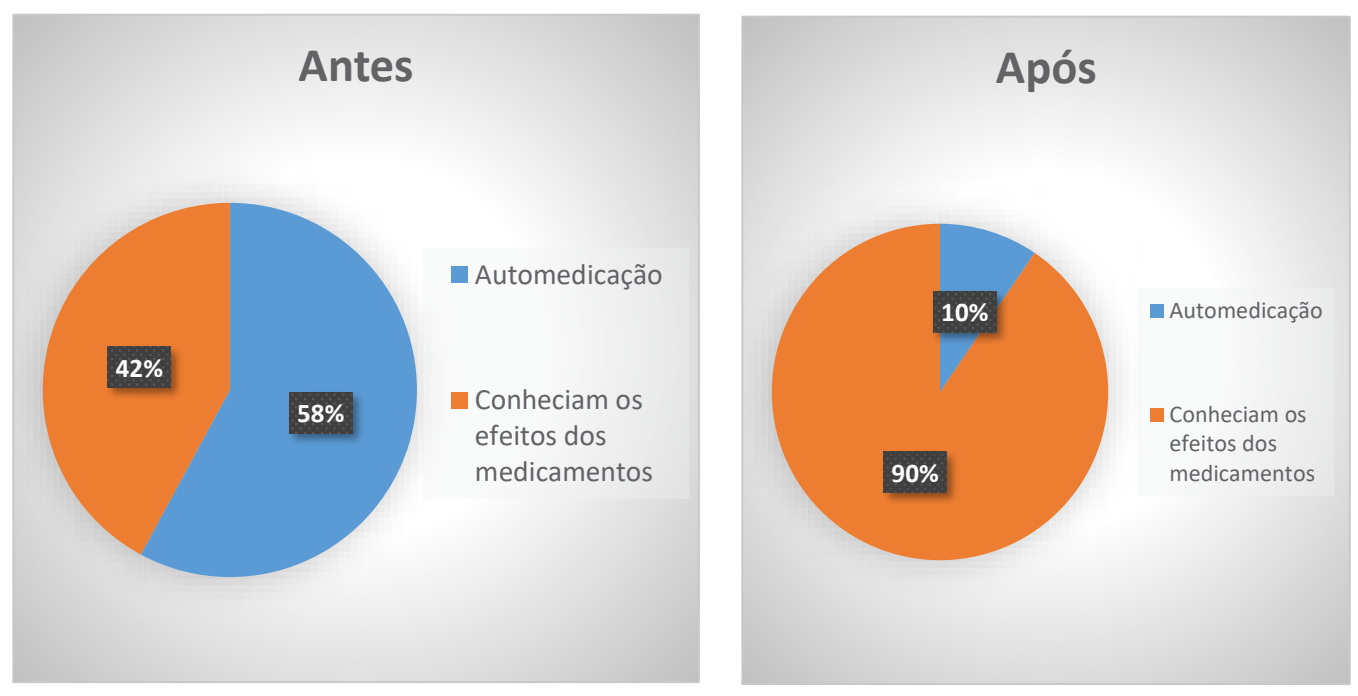
Entre as perspectivas sobre o projeto de extensão SAFI, afirmadas pelos participantes esta a influência do projeto na vida deles que foi relatada por $17(81 \%)$ idosos. As orientações e palestras ministradas no projeto contribuíram na vida de 18 (86\%) idosos. Cerca de 16 (76\%) idosos fazem o uso dos conhecimentos adquiridos no SAFI no seu cotidiano. Com base nas atividades de educação e saúde realizadas semanalmente foram sendo explicados alguns termos sobre medicamentos para os idosos. Esses termos foram questionados durante a entrevista para avaliar se os pacientes tinham a compreensão da sua farmacoterapia. A tabela 2 descreve a percepção dos idosos quanto os termos questionados.

Tabela 2 - Percepção dos idosos quanto os termos relacionados a farmacoterapia, Remanso da Paz, Quixadá (CE), 2016.

\begin{tabular}{c|c|c}
\hline Termos & Frequência & (\%) \\
\hline “Interação medicamentosa” & 3 & $14 \%$ \\
\hline “Efeito adverso" & 2 & $10 \%$ \\
\hline "Sobredosagem" & 7 & $33 \%$ \\
\hline “Automedicação" & 7 & $33 \%$ \\
\hline
\end{tabular}

A tabela 3 descreve dados sobre o uso de medicamentos e plantas medicinais, como também as noções sobre a automedicação.

Tabela 3 - Questionário realizado com idosos na casa de acolhida Remanso da Paz, Quixadá (CE), 2016.

\begin{tabular}{c|c|c}
\hline Entrevista feita com os idosos do projeto de extensão SAFI & Frequência & (\%) \\
\hline Faz o uso correto do medicamento & 19 & $90 \%$ \\
\hline Utiliza a mesma posologia prescrita pelo médico & 21 & $100 \%$ \\
\hline Já sentiu algum mal-estar após tomar algum medicamento & 3 & $14 \%$ \\
\hline Teve alguma reação alérgica & 4 & $48 \%$ \\
\hline Tem noção dos riscos da automedicação & 10 & $52 \%$ \\
\hline Faz uso de preparações medicinais & 11 & \\
\hline Tem conhecimento de que o uso de plantas medicinais pode haver \\
interação com seus medicamentos
\end{tabular}

\section{Discussão}

Atualmente, o uso de medicamentos pelos idosos tem gerado preocupação quanto aos gastos excessivos no sistema de saúde e aos possíveis efeitos, benéficos ou indesejáveis. O perfil de uso obedece às peculiaridades de idade, gênero, inserção social, estado de saúde e classe terapêutica 9 .

Flores \& Mengue ${ }^{10}$ avaliaram idosos, sendo 141 (66\%) mulheres e $117(54 \%)$ homens, na faixa etária entre 60 e 70 anos. A prevalência de uso de medicamentos foi de $91 \%(n=195)$. Na semana anterior à entrevista foram utilizados 697 medicamentos, com média de 3,2 ( $\mathrm{DP}=2,5)$ medicamentos por pessoa. Do total da amostra, $187(87 \%)$ haviam realizado no mínimo uma consulta médica no último ano, 71 (33\%) pessoas usavam medicamento sem prescrição médica e em 57 $(27 \%)$ casos foi caracterizada polifarmácia. 
No presente estudo a população idosa, houve maior predominância de mulheres 13 (62\%), idosos viúvos 13 (62\%) e que habitavam em casa própria $15(72 \%)$ com faixa etária de 67 a 91 anos. Evidencia-se quanto ao sexo, que, as mulheres são mais propensas a doenças, e quando a isso, maior probabilidade de uso de medicamentos. Em relação ao sexo, dados semelhantes foram encontrados no estudo de Oliveira et al. ${ }^{11}$, onde à população estudada eram em maior predominância mulheres $866(57,2 \%)$ e que a baixa escolaridade dos idosos foi considerada um importante fator de risco diante da complexidade dos esquemas medicamentosos utilizados pelos entrevistados, à medida que se constatou que $1,032(68,1 \%)$ são analfabetos ou tiveram pouco tempo de escolaridade, já em nosso estudo foi visto que $11(53 \%)$ dos idosos entrevistados tinha concluído $01^{\circ}$ grau e apenas $4(19 \%)$ eram analfabetos o que mostra baixa prevalência de escolaridade comparado com este estudo.

Oliveira \& Araujo ${ }^{12}$ descreve que a maior incidência do sexo feminino deve-se ao fato de uma maior participação das mulheres no grupo de idosos pesquisado, sugerindo que as idosas aparentam um maior interesse em participar de atividades educativas e de lazer do que o idoso, ou ainda que, mesmo após a aposentadoria, os homens procuram exercer alguma profissão, preferindo dedicar seu tempo livre a alguma atividade remunerada, sobrando menos tempo para se dedicar a atividades de lazer e aos recursos da comunidade.

Em um estudo realizado em Porto Alegre, Rio Grande do Sul, 182 (60,5\%) afirmaram não esquecem de tomar os medicamentos, faziam uso de medicamentos por conta própria 111 (36,9\%) idosos e, além disso, $225(74,5 \%)$ afirmam ter recebido orientação quanto ao uso do medicamento e $246(81,7 \%)$ afirmam que sabem a indicação do mesmo, enquanto apenas $82(27,2 \%)$ dizem estar informados sobre os efeitos adversos dos medicamentos que utilizam ${ }^{3}$.

A educação em saúde baseia-se no diálogo e na troca de conhecimentos, associando-se entre o saber científico e o popular, tendo em vista que os processos comunicativos se baseiam em escutar o outro para compreender quais as suas crenças, sua situação e suas possibilidades de poder atuar conjuntamente. Portanto, a comunicação na atenção à saúde do idoso é algo que se constrói em uma ação intencional, dirigida e orientada para um único interesse ${ }^{6}$.

Observou-se que as ações de educação em saúde desenvolvidas através do projeto SAFI, incentivaram os idosos a minimização da prática da automedicação. Foram observadas influências positivas na vida pessoal dos idosos que participaram efetivamente dos encontros $17(81 \%)$ e as informações obtidas pelas palestras foram utilizadas na vida diária de 16 (76\%) dos mesmos.

Luz, Lima \& Monteiro (2013) ${ }^{13}$ em uma pesquisa com 100 indivíduos de idade superior a 60 anos, 69 (69\%) afirmaram ter conhecimentos sobre os medicamentos que usavam. Ainda, foi visto que os participantes buscam informações aos farmacêuticos, quando vão procurar os medicamentos e aos médicos no momento da consulta. Normalmente, não são informados sobre os efeitos adversos e muito menos sobre as interações medicamentosas que podem ocorrer com os medicamentos. Os autores ainda afirmaram que alguns idosos usam muitos medicamentos ao mesmo tempo e não estão prevenidos sobre os riscos disso, ou seja, não são alertados sobre como prevenir possíveis interações.

Andrade, Silva \& Freitas ${ }^{14}$ afirmam que o profissional farmacêutico está mais próximo nessa atuação, esclarecendo informações sobre a posologia, interações com alimentos e/ou medicamentos, reações adversas e alérgicas e os perigos da automedicação, tendo em vista melhorar a qualidade de vida de cada paciente por meio de resultados definidos na terapia medicamentosa.

Peretta \& Ciccia ${ }^{15}$ relatam que os problemas que costumam ocorrer no uso de medicamentos em idosos vão desde a escolha inadequada do medicamento, falha ao receber o medicamento, uso inadequado (esquecimento), dose subterapêutica, superdosagem, efeitos adversos, interações farmacológicas e automedicação.

Mochel et al. ${ }^{16}$ em entrevista com 462 idosos foi visto que 335 (75\%) pacientes afirmaram que tomavam a medicação de acordo com a prescrição médica e que $111(25 \%)$ pacientes diziam que não tomavam a medicação de acordo com 0 que havia sido prescrito. Diferindo do presente estudo, o qual $19(90 \%)$ afirmaram usar corretamente os medicamentos, e $21(100 \%)$ afirmaram seguir a mesma posologia prescrita pelo médico, o que reflete positivamente na conduta clínica do estado de saúde dos idosos, que por serem uma população com maior risco de comorbidades, na maioria das vezes esse cuidado e atenção a farmacoterapia não é realizado.

Oliveira et al. ${ }^{11}$, entre os idosos que utilizavam medicamentos, $1.116(91,1 \%)$ relataram ter consumido fármacos exclusivamente prescritos por médicos ou dentistas e 106 (8,9\%; IC95\%: 6,7- 11,5) referiram consumo simultâneo de medicamentos prescritos e não prescritos ${ }^{11}$. O presente estudo apresenta que (10) $48 \%$ dos participantes afirmaram ter noção dos riscos da automedicação. 
Na pesquisa de Luz, Lima \& Monteiro ${ }^{13}$ o conhecimento das expressões foi visto que 4 (4\%) de indivíduos que conheciam o termo "Interação Medicamentosa", 94 (94\%) que não a conheciam e 2 (2\%) não responderam; para a expressão "Efeito Secundário" 7 (7\%) a conheciam, 91 (91\%) não a conheciam e 2 (2\%) não responderam; relativamente à "Sobredosagem" $10(10 \%)$ a conheciam, 88 (88\%) não sabem o significado e $2(2 \%)$ não responderam; "Efeitos Adversos" 8 (8\%) afirmaram ter conhecimento do significado, 90 (90\%) não conheciam o significado e $2(2 \%)$ não responderam; para a "Automedicação" $9(9 \%)$ teve conhecimentos do significado, 88 (88\%) não sabem o significado e $3(3 \%)$ não responderam. Os autores ainda revelaram uma necessidade de profissionais disponibilizar as devidas informações aos idosos para que esses consigam desenvolver o tratamento medicamentoso de forma efetiva.

No presente estudo na avaliação da compreensão da farmacoterapia sobre os termos questionados, percebe-se uma lacuna exposta na ausência de conhecimento das expressões. Sobre o termo "Interação medicamentosa" apenas $3(14 \%)$ afirmavam conhece-lo, o que evidencia maiores riscos de fatores que possam influenciar na terapia, como os alimentos. Apenas $2(10 \%)$ conheciam o que era "Efeito adverso" e $7(33 \%)$ relataram saber o que era a expressão "Sobredosagem" o que por vezes, o paciente pode apresentar algum efeito negativo relacionado ao uso dos medicamentos, mas dificulta sua interpretação da real causa. Por fim, 7 (33\%) conheciam sobre a prática da "Automedicação", o que eleva a probabilidade de agravos á saúde dos idosos.

Os principais fatores que possibilitam a automedicação, de acordo com Bortolon, Karnikowski \& Assis ${ }^{17}$, são a falta e /ou o desinteresse pela busca da informação, o mínimo empenho das identidades responsáveis na disponibilização das informações, a presença de estoque de medicamento nas residências e a globalização.

Uma das consequências relacionadas com o uso de medicamentos sem prescrições são as reações alérgicas que por vezes surgem com alguns indivíduos, devidas as distintas características individuais. Luz, Lima \& Monteiro (2013) ${ }^{13}$ verificaram que $86(86 \%)$ afirmaram que nunca tiveram nenhuma reação alérgica a qualquer medicamento, mas 10 (10\%) afirmam já ter tido reações, essencialmente com as penicilinas e, 4 (4\%) do total não responderam ou não sabem se já tiveram ou não interações ${ }^{11}$.

A utilização de plantas medicinais pode trazer riscos relacionados à qualidade de vida e à segurança, já que no ato de obtenção pode ocorrer a identificação errônea da planta. Uma das estratégias em serviços de atenção básica à saúde para atenuar esse risco seria a adoção de hortos de plantas medicinais, cujas espécies seriam certificadas por botânicos, e sua indicação e uso ficariam sob a responsabilidade de equipe de saúde multiprofissional ${ }^{12}$.

Assim, o emprego de plantas medicinais, apesar de constatado, parece ser ignorado pelos profissionais das equipes de saúde, visto que os homens idosos relataram não ter recebido orientações sobre possíveis riscos em relação ao uso conjunto com os medicamentos industrializados. A fonte de informações sobre plantas medicinais geralmente são amigos e parentes, paralelamente a consultas feitas a livros que possuíam em casa, sem, no entanto, atentar para questões relacionadas à dose da planta, complicações ou intoxicações decorrentes, levando a falta de conhecimento sobre interações entre medicamentos e plantas e que as consequências dessas associações podem interferir na eficácia dos medicamentos utilizados, principalmente, para tratar doenças crônicas, como a hipertensão e 0 diabetes ${ }^{18}$.

$O$ profissional Farmacêutico tem a capacidade de orientar a respeito dos medicamentos prescritos e dispensados aos idosos, pois eles estão em contato frequente com os pacientes discutindo sobre os problemas de saúde, informando sobre a natureza da doença crônica e identificando as razões do tratamento. Além de educar quanto aos riscos da automedicação, as interações em idosos polimedicados e assim permitindo uma eficácia na terapêutica.

Cabe ressaltar que esta pesquisa foi realizada através da coleta de informações envolvendo a lembrança dos idosos, o que pode levar a subjetividade dos dados, pois, a recordação de fatos passados, de certa forma, pode não corresponder totalmente com a realidade.

\section{Considerações Finais}

Os resultados do estudo mostram baixa prevalência de automedicação entre os idosos e apontam a partir das atividades de educação em saúde, houve influência positivas nesses pacientes. Em relação ao fenômeno em estudo foi possível notar a ausência de conhecimento de algumas expressões para a compreensão das informações sobre os medicamentos, tais como efeito secundário, interação medicamentosa, efeito adverso, etc., mas uma proporção significativa 10 (48\%) tem 0 discernimento dos riscos advindos da automedicação. Ressalta-se que 21 (100\%) dos entrevistados demonstrou utilizar os medicamentos de acordo com a prescrição, o que reflete positivamente no manejo clínico do idoso. 
Observa-se a intervenção positiva da atuação do projeto SAFI na vida dos idosos, e a crença de que a atenção farmacêutica fortalece a adesão da farmacoterapia, possibilitando a transmissão de informações sobre as doenças e o uso racional de medicamentos, e com isso ver-se a necessidade de desenvolver programas com fins de minimizar 0 consume de medicamentos sem conhecimento prévio pela população idosa.

\section{Referências}

1. Brasil. Ministério da Saúde. Secretaria de Atenção à Saúde. Departamento de Atenção Básica. Envelhecimento e saúde da pessoa idosa / Ministério da Saúde, Secretaria de Atenção à Saúde, Departamento de Atenção Básica. Brasília: Ministério da Saúde, 2007.

2. Silva CAM, Almeida A. A importância da família no cuidado ao idoso. 2012; 6(6):1-12.

3. Marin JS, Cecílio LCO, PerezAEWU, Santella F, Silva CBA, Filho JRG, Roceti LC. Caracterização do uso de medicamentos entre idosos de uma unidade do Programa Saúde da Família. Cad. Saúde Pública, Rio de Janeiro. 2008; 24(7).

4. Marcieni AA, Marcos VSS, Osvaldo F. Assistência Farmacêutica como Estratégia para o Uso Racional de Medicamentos em Idosos. 2004; 25(1).

5. Cordeiro BC, Leite SN. O Farmacêutico na atenção à saúde. Itajaí: Universidade do Vale do Itajaí. 2005: 189 p.

6. Santos SLF, Almeida RO, Paiva CEQ, Barros KBNT, Arraes MLBM. Serviço de Atendimento Farmacêutico ao Idoso: relato de experiência de educação em saúde. Rev Santa Maria. 2016; 42(2):02-07.

7. Brites R. Manual de Técnicas e Métodos Quantitativos Tomo - I. INA - Instituto Nacional de Administração. Lisboa, Junho de 2007.

8. Brasil. Ministério da Saúde. Conselho Nacional de Saúde. Diretrizes e normas regulamentadoras sobre pesquisa envolvendo seres humanos. Resolução 466.2012. Brasília: CNS; 2012.

9. Rozenfeld S. Prevalência, fatores associados e mau uso de medicamentos entre os idosos: uma revisão. Cad. Saúde Pública, Rio de Janeiro. 2003; 19(3):717-724.

10. Flores LM, Mengue SS. Uso de medicamentos por idosos em região do sul do Brasil. Rev. Saúde Pública, São Paulo. 2005; 39(6):924-929.

11. Oliveira MA, Francisco PMSB, Costa KS, Barros MBA. Automedicação em idosos residentes em Campinas, São Paulo, Brasil: prevalência e fatores associados. Cad. Saúde Pública, Rio de Janeiro. 2012; 28(2):335-345.

12. Oliveira CJ, Araujo TL. Plantas medicinais: usos e crenças de idosos portadores de hipertensão arterial. Revista Eletrônica de Enfermagem. 2007; 9(1):93-105.

13. Luz DJ, Lima JAS, Monteiro LG. Automedicação no Idoso. Universidade do Mindelo. 2013: 10-60 p.

14. Andrade MA, Silva MVS, Freitas O. Assistência Farmacêutica como Estratégia para o Uso Racional de Medicamentos em Idosos. Semina: Ciências Biológicas e da Saúde, Londrina. 2004; 25(1):55-64.

15. Peretta M, Ciccia G. Reengenharia farmacêutica - guia para implantar atenção farmacêutica. Brasília: Ethosfarma. 2000: 45-64 p.

16. Mochel EG, Andrade CF, Almeida DS, Tobias AF, Cabral R, Cossetti RD. Avaliação do tratamento e controle da hipertensão arterial sistêmica em pacientes da rede pública em São Luis (MA). Revista Baiana de Saúde Pública. 2007; 31(1):90-101.

17. Bortolon PC, Karnikowski MGO, Assis M. Automedicação versus indicação farmacêutica: O profissional de farmácia na atenção primária à saúde do idoso. Revista APS. 2007; 10(2):200-209.

18. Lima SCS, Arruda GO, Renovato RD, Alvarenga MRM. Representações e usos de plantas medicinais por homens idosos. Rev. Latino-Am. Enfermagem, Ribeirão Preto. 2012; 20(4). 


\section{Hérick Hebert da Silva Alves}

Endereço para correspondência - Rua: Tabelião Enéas de Lima, $n^{\circ} 171$,

Bairro: Centro, CEP: 63900-065,Quixadá, CE, Brasil.

E-mail: herick_hebert@hotmail.com

Lattes: http://lattes.cnpq.br/1186584354920031

Sanny Ellen de Souza Pereira - sannyellenpereira@hotmail.com

Sandna Larissa Freitas dos Santos - sandy.lary@hotmail.com

Karla Bruna Nogueira Torres Barros - karlabruna1@hotmail.com

Maria Luísa Bezerra de Macedo Arraes - mariaarraes@fcrs.edu.br

Enviado em 18 de outubro de 2016. Aceito em 30 de dezembro de 2016. 